\title{
Even cognitively well-functioning adults are unaware of their olfactory dysfunction: Implications for ENT clinicians and researchers*
}

\author{
Eike Wehling 1,2, Astri J. Lundervold 1,2, Thomas Espeset ${ }^{3,4}$, Ivar Reinvang33, \\ Annika Brämerson ${ }^{5}$, Steven Nordin 6 \\ Rhinology 53: 89-94, 2015 \\ DOI:10.4193/Rhino14.081 \\ 1 Department of Biological and Medical Psychology, University of Bergen, Norway \\ *Received for publication: \\ ${ }^{2}$ Kavli Research Centre for Aging and Dementia, Haraldsplass Hospital, Bergen, Norway \\ March 23, 2014 \\ ${ }^{3}$ Department of Psychology, University of Oslo, Norway \\ Accepted: October 9, 2014 \\ ${ }^{4}$ Norwegian Centre for Mental Disorders Research (NORMENT), and KG Jebsen Centre for Psychosis Research, Division of Mental \\ Health and Addiction, Oslo University Hospital, Norway \\ ${ }^{5}$ Department of Otorhinolaryngology, Central Hospital, Skövde, Sweden \\ ${ }^{6}$ Department of Psychology, Umeå University, Sweden
}

\begin{abstract}
Background: Past findings of an impact of cognitive impairment on awareness of olfactory dysfunction, and high prevalence of age-associated cognitive impairment motivated the present study of whether middle-aged and elderly adults are unaware of an olfactory dysfunction despite being carefully screened for cognitive impairment.
\end{abstract}

Methodology: The sample included 203 Norwegian participants, aged 46-79 years, 134 women and 69 men, who underwent comprehensive neuropsychological assessment for screening of cognitive impairment. Subjective assessment of olfactory function ("How would you estimate your sense of smell?") was compared with outcome on objective assessment of olfactory function with the Scandinavian Odor Identification Test, which in the present study was shown to be valid for use on Norwegian populations.

Results: We found that $79 \%$ of this cognitively healthy sample with objectively assessed olfactory dysfunction reported normal olfactory function ( $57 \%$ of functionally anosmics reported normal function). In contrast, only $9 \%$ with objectively assessed normal olfactory function reported olfactory dysfunction.

Conclusion: A large proportion of cognitively well-functioning middle-aged and elderly adults with an olfactory dysfunction are unaware of their dysfunction. The ENT physician who suspects that the sense of smell may be compromised should, in addition to an anamnesis, assess the patient's olfactory function objectively.

Key words: aging, olfaction, self-evaluation, unawareness, odour identification

\section{Introduction}

Olfaction can be considered the oldest sensory system from an evolutionary perspective. Therefore, its role and function differs significantly from that of the relatively young senses, such as vision and audition. Humans have inherited the basic function of olfaction from very primitive organisms, such that the odour perception from a substance shall either attract us to that substance or make us avoid it, depending on positive or negative as- sociations established at prior interactions with the substance ${ }^{(1)}$. Since this function was present among very primitive organisms, in which behaviour was largely controlled by instinct, awareness of odour perception is not a prerequisite for olfaction to fulfill its role. Thus, we are typically unconscious of the continuous monitoring we do of the chemical air quality surrounding us, and about the guiding impact that odorous substances have on our behaviour. An advantage of this is that our attention can instead 
be directed towards other aspects that we encounter. However, since awareness is not a prerequisite for successful monitoring, this characteristic feature of olfaction may also result in us not being aware of a gradual loss in olfaction.

The role of conscious evaluation of one's ability to smell is illustrated by results from a study in which healthy, fairly young subjects rated their olfactory function, followed by objective testing of the function. In a second group of subjects, the order of assessment was reversed. A significant correlation was obtained between the subjective and objective evaluation when the objective evaluation was performed first, but not when the subjective evaluation was performed first. From this the authors concluded that ratings of olfactory function may become more accurate after having experienced smell assessment ${ }^{(2)}$.

Unawareness of olfactory dysfunction has been shown to be particularly common in individuals with cognitive impairment, including Alzheimer's disease ${ }^{(3,4)}$ and mild cognitive impairment (5). Some authors have even suggested that unawareness could serve as a predictor for Alzheimer's disease ${ }^{(6)}$. Furthermore, epidemiological studies show that unawareness of an age-related gradual loss in olfaction is common. In addition to an exponential increase in olfactory loss with age, results from a Swedish population-based adult sample aged $18-80+$ years, in which test odorants were used to assess olfactory function, showed that $13 \%$ were hyposmic (reduced sense of smell) and $6 \%$ were functionally anosmic (complete loss of smell). However, only $44 \%$ of those with anosmia or hyposmia were actually aware of having an olfactory dysfunction ${ }^{(7)}$. In a similar study in the US of adults aged 53-97 years, 24\% were either functionally anosmic or hyposmic, and only $20 \%$ of those were aware of having a dysfunction ${ }^{(8)}$. Similar findings of unawareness of olfactory dysfunction have been reported in clinical studies $(4,9,10,11)$. This evokes the question whether the ENT physician can rely on selfreports of olfactory function even if the patient is cognitively well-functioning.

About $20 \%$ of individuals in the general elderly population show cognitive impairment ${ }^{(12)}$. Still, such impairment is commonly not controlled for in prior studies of awareness of olfactory dysfunction in elderly. It is thus not settled to what extent unawareness of dysfunction reported in this age group can be explained by cognitively non-healthy participants being included and affecting the results. To address this issue, the objective of the current study was to examine awareness of olfactory dysfunction and normal olfactory function in cognitively healthy middleaged and older adults who were carefully screened for cognitive impairment by means of a comprehensive neuropsychological examination.

Since awareness of the odorous substance is not a prerequisite for olfaction to fulfill its role, as reviewed above, it was hypothesized that unawareness of olfactory dysfunction would be common also among cognitively well-functioning adults. Consequences of olfactory dysfunction include risk for inappropriate food choice, poor appetite, mood changes and depressive symptoms, feelings of vulnerability, and impact on social interaction, resulting in reduced quality of life ${ }^{(13,14)}$. Considering that olfaction is an important chemical warning system, an olfactory dysfunction that the individual is not aware of may have serious consequences for safety. Not being aware of this warning system being "turned off" may result in not taking necessary precautions when not being able to perceive, for example, hazardous chemical exposure, spoiled food and fire. This implies that simply asking a patient about his/her olfactory status, when the person has a health condition that is likely to result in an olfactory dysfunction, may thus be seriously insufficient. Although complete clinical assessment of olfactory capabilities or use of at least one standardized quantitative measure of olfactory function has been proposed ${ }^{(15)}$, this seems not to have reached routine status since such assessment is rarely part of medical examination.

The Scandinavian Odor Identification Test (SOIT) was used to objectively assess olfactory function in a Norwegian sample. Since development, evaluation and normative data of the SOIT have been based on Swedish participants only, we also investigated the validity of the SOIT for use in Norway. This was conducted by comparing the Norwegian sample with a Swedish sample with respect to distribution of diagnoses across normal olfactory function and olfactory dysfunction (based on the SOIT) and by investigating the discriminative validity of the SOIT in terms of its ability to detect age-related differences in a Norwegian sample by comparing three age groups. Due to an expected exponential increase in olfactory dysfunction with age, it was expected that the strongest age-related effect would be found when comparing the youngest and oldest age groups.

\section{Materials and methods}

\section{Participants}

Two-hundred and forty-seven persons, aged 45-79 years, participated, for whom complete cognitive data and objectively and subjectively assessed olfactory data were collected. The participants took part in a study of cognitive aging in Oslo and Bergen, Norway, in which past or present neurological and psychiatric diagnoses were exclusion criteria. The main Norwegian project was approved by the Regional Committee for Research Ethics of Southern Norway. The Skövde Population-Based Study, from which data was used to assess metric properties of the SOIT for Norwegian use, was approved by the Ethics Committee at the University of Gothenburg (Gothenburg, Sweden). The studies were performed according to the Declaration of Helsinki on 
guidelines for biomedical research involving human subjects ${ }^{(16)}$. All participants gave their informed consent.

The sample underwent comprehensive neuropsychological assessment including tests of general cognitive ability (Vocabulary and Matrices subscales of the Wechsler Abbreviated Scale of Intelligence, WASI) ${ }^{(17)}$, verbal learning and memory function (California Verbal Learning Test) ${ }^{(18)}$, executive functioning and attention (Color Word Interference Test; Trail Making Tests A and B) (19-21), and mental processing speed (Digit Symbol Test, Wechsler Adult Intelligence Scale-R) ${ }^{(22)}$. The cognitive profile of each participant was examined for possible impairment. Inclusion criteria in the present study were (i) an IQ score $>85$ according to two subtests of the WASI ${ }^{(17)}$ (excluding 6 participants), (ii) an ageand gender-adjusted score > -1.5 SD on the verbal memory task, commonly used to define mild cognitive impairment ${ }^{(23)}$ (excluding 4 participants), and (iii) at the maximum one performance score that was 1 SD below age-adjusted peer normative data on any of the neuropsychological tasks (excluding 34 participants). Hence, the final sample consisted of 203 persons, aged 46-79 years ( mean $=62.8, S D=8.0$ ), of whom 134 were women and 69 were men. Mean scores on the neuropsychological measures in the sample are presented in Table 1.

\section{Objective assessment of olfactory function}

The SOIT ${ }^{(24)}$ was used to objectively assess olfactory function. It consists of 16 odorous stimuli: pine-needle, peppermint, juniper berry, violet, anise, clove, vanilla, almond (bitter), orange, cinnamon, lemon, lilac, vinegar, tar, ammonia, and apple. The stimulus order was randomized for each participant. Ammonia $(1.0 \mathrm{~mol} / \mathrm{L}), \mathrm{tar}$, and vinegar were natural products, whereas the remaining stimuli were natural oils (Stockholm Ether \& Essence Manufactory, Stockholm, Sweden). The liquid odorant was injected into a tampon filled to saturation and placed in an opaque glass jar. For each stimulus the participant was provided with a written list of the four response alternatives from which to choose the most appropriate item for identification ${ }^{(24)}$. The stimuli were presented birhinally, about $5 \mathrm{~cm}$ under the participant's nose with an inter-stimulus interval of about $20 \mathrm{~s}$ to avoid adaptation. No time restrictions were given for the participants to make their choice. Testing was conducted in a well-ventilated room. The SOIT has good test-retest reliability, split-half reliability and validity for Swedish populations ${ }^{(24)}$. A validation of the SOIT for use of Norwegian populations was conducted, and is described in the Appendix.

\section{Subjective assessment of olfactory function}

Prior to the olfactory testing the participants were asked to evaluate their sense of smell with the question "How would you estimate your sense of smell?"The response alternatives were "better than normal", "normal", and "poorer than normal." For the
Table 1. Neuropsychological measures.

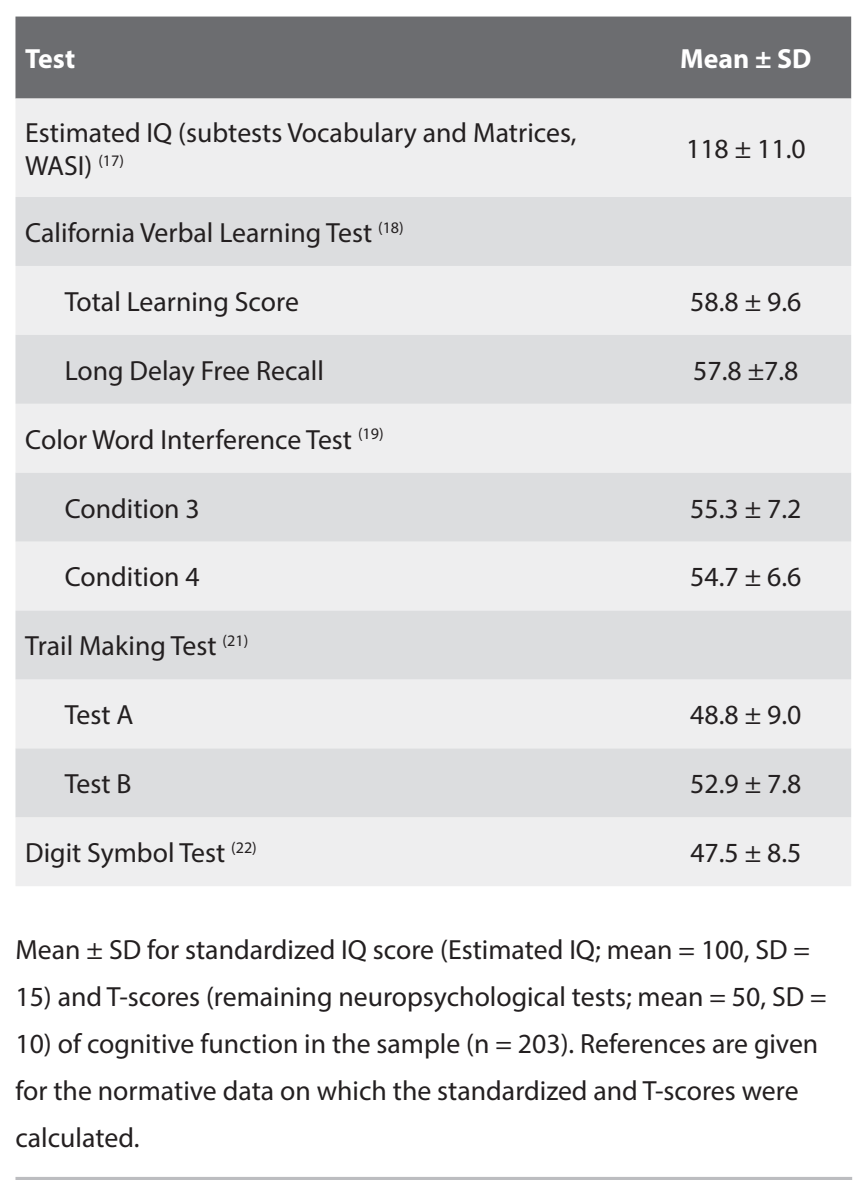

current purpose, the first two response alternatives were referred to as subjectively assessed normal olfactory function and the third alternative was referred to as olfactory dysfunction.

\section{Results}

The number of participants with objectively and subjectively assessed normal olfactory function and olfactory dysfunction are presented in Table 2. The categorization of normal olfactory function and olfactory dysfunction (hyposmia or functional anosmia) for the objective assessment was based on diagnostic cut-off scores for the SOIT for young adults (15-34 years) ${ }^{\text {(24). }}$ The results showed that $28 \%$ of the sample had an objectively assessed olfactory dysfunction, and $12 \%$ had a subjectively assessed olfactory dysfunction. The distribution across diagnoses (Table 4) was significantly different between the objective and subjective assessments $\left[x^{2}(1)=6.745, p<0.01\right]$. The sensitivity of the subjective assessment (using the objective assessment for comparison) was $21 \%$ and the specificity was $91 \%$. Thus, $79 \%$ of this cognitively healthy sample who had an objectively assessed olfactory dysfunction reported normal olfactory function. This percentage was 82 among hyposmics and 57 among functionally anosmics. The overall correct classification rate was $71 \%$. Thus, the results show that unawareness of olfactory dysfunction is common in cognitively well-functioning middle-aged and 
Table 2. Frequencies of cognitively healthy $(n=203)$ participants with objectively and subjectively assessed normal olfactory function and olfactory dysfunction.

\begin{tabular}{|lccc|}
\hline Objective assessment \\
\hline Subjective assessment & $\begin{array}{c}\text { Normal } \\
\text { olfactory } \\
\text { function }\end{array}$ & $\begin{array}{c}\text { Olfactory dysfunc- } \\
\text { tion (anosmic/ } \\
\text { hyposmic) }\end{array}$ & All \\
\hline Normal olfactory function & 133 & $45(41 / 4)$ & 178 \\
\hline Olfactory dysfunction & 13 & $12(9 / 3)$ & 25 \\
\hline All & 146 & $57(50 / 7)$ & 203 \\
\hline
\end{tabular}

old adults.

\section{Discussion}

The fact that we, despite continuous use of our sense of smell, do not need to pay continuous attention to odorous substances in our environment provided the basis for the present hypothesis stating that even cognitively well-functioning individuals typically are unaware of an olfactory dysfunction. This was investigated by comparing self-reports of olfactory function with objectively assessed olfactory function in a Norwegian sample aged 46-79 years. The SOIT and its cut-off scores were used for the objective assessment of olfactory function, which in the present study were found to be valid for use also in Norway. The careful screening for cognitive impairment by means of comprehensive neuropsychological assessment suggests that the sample of middle-aged and elderly were cognitively healthy. The results do indeed support the hypotheses that unawareness of olfactory dysfunction is common also among cognitively healthy adults. Thus, $79 \%$ of the cognitively healthy participants who had an objectively assessed olfactory dysfunction were unaware of their dysfunction. Unawareness was common (57\%) even among the functionally anosmic participants. In contrast to the poor awareness of a dysfunction, the specificity of $91 \%$ suggests that a large majority of cognitively healthy middleaged and elderly persons who have a normal functioning sense of smell are aware of this normality.

With a sensitivity and specificity of $21 \%$ and $91 \%$, respectively, the results of this study agree well with the sensitivity (20\%) and specificity (94\%) reported by Murphy et al. ${ }^{(8)}$, and the specificity (85\%) reported by Brämerson et al. ${ }^{(7)}$. However, the sensitivity in the latter study was higher (44\%) than in the present study. This discrepancy may be due to the question in that study being pertained to odour detection sensitivity rather than to the more general question of estimating the sense of smell, as in the present study.
An important implication of the results for the ENT physician, or any other examiner, is that it is not sufficient to simply ask the patient whether he/she has an olfactory dysfunction when the patient has a medical condition that may affect olfaction. Over 200 aetiologies for olfactory dysfunction have been listed ${ }^{(25)}$, with underlying mechanisms that can be classified as conductive (e.g., polyposis), sensorineural (e.g., post-URI) or impairment in the olfactory central nervous system (e.g., tumour). Not knowing that one cannot perceive, for example, hazardous chemical exposure, spoiled food and fire may have serious consequences for the patient's safety ${ }^{(13,14)}$. Even in cases when there is no treatment for the olfactory dysfunction the patient can, if becoming aware of the dysfunction, benefit from coping strategies and social support ${ }^{(26)}$.

The unawareness may be particularly common when the loss in functionality is gradual over time, such as in aging. Notably, the oldest segment of the population will increase within the next decade, and thereby the prevalence of age-related olfactory dysfunction.

Several tests of the ability of odour identification are available. Due to the need to use culturally relevant test odorants to assess odour identification, tests for different geographical regions have been developed with demonstrated validity and reliability, and with normative data. Such regions include the USA ${ }^{(27,28)}$, Central Europe (29), Scandinavia ${ }^{(24)}$, and Japan ${ }^{(30)}$. In addition to odour identification, the Sniffin' Sticks can be used to separately assess olfactory detection and discrimination ${ }^{(29)}$. If time restriction is a concern in the clinical routine, there are also very short versions of tests of odour identification that can be used, with fairly good metric properties ${ }^{(31-33)}$.

In addition to loss in the ability to identify odorous substances, which was the focus of the present study, it is rather common that the patient may be seeking medical attention for parosmia (a qualitative odour distortion) and/or phantosmia (an odour sensation in the absence of an external odorant). At date there are no objective methods available for investigation of these conditions. Instead we are limited to the use of self-reports. For these purposes either a short ${ }^{(34)}$ or a more extensive ${ }^{(35)}$ questionnaire instrument can be used.

In conclusion, the results from the present study suggest that even cognitively healthy middle-aged and elderly adults are unaware of their olfactory dysfunction. The ENT physician and other clinicans who suspects that the sense of smell may be compromised should, in addition to an anamnesis, assess the patient's olfactory function with objective tests. 


\section{Acknowledgements}

The study was supported by grants from the Regional Health Authorities in Bergen (Helse Bergen) to Eike Wehling (grant 911461), and from the Swedish Research Council for Health, Working Life and Welfare to Steven Nordin (grant 2011-0396). We would like to thank the test technicians at the Neuropsychological Outpatient Clinic, Department of Biological and Medical Psychology, University of Bergen for their technical support, and professor Mats Bende for giving us access to data from the Skövde Population-Based Study.

\section{Authorship contribution}

Eike Wehling (EW), Astri J. Lundervold (AJL), Thomas Espeset (TE), Ivar Reinvan (IR) and Steven Nordin (SN) contributed to the design of the main Norwegian study, and Annika Brämerson $(A B)$ and SN to the design of the Skövde Population-Based Study (from which data was used to assess metric properties of the SOIT for Norwegian use). For the main Norwegian study, EW was responsible for the data collection, and EW and SN analysed the data and interpreted the results. EW and SN wrote most of the manuscript, with contributions from $A J L, T E, I R$ and $A B$.

\section{Conflict of interest}

The authors declare that there is no conflict of interest.

\section{References}

1. Engen T. Odor sensation and memory. New York: Greenwood Publishing Group, 1991

2. Landis BN, Hummel T, Hugentobler M, Giger R, Lacroix JS. Ratings of overall olfactory function. Chem Sens. 2003; 28: 691-694.

3. Doty RL, Reyes PF, Gregor T. Presence of both odor identification and detection deficits in Alzheimer's disease. Brain Res Bull. 1987; 18: 597-600.

4. Nordin S, Monsch A, Murphy C Unawareness of smell loss in normal aging and Alzheimer's disease: discrepancy between self-reported and diagnosed smel sensitivity. J Gerontol. 1995; 50B: 187-192.

5. Tabert, MH, Albert SM, Borukhova-Milov $L$, et al. Functional deficits in patients with mild cognitive impairment: prediction of AD. Neurology. 2002; 58: 758-764.

6. Devanand DP, Michaels-Marston KS, Liu X, et al. Olfactory deficits in patients with mild cognitive impairment predict Alzheimer's disease at follow-up. Am J Psychiat. 2000; 157: 1399-1405.

7. Brämerson A, Johansson L, Ek L, Nordin, S, Bende M. Prevalence of olfactory dysfunction: the Skövde population-based study. Laryngoscope. 2004; 114: 733-7.

8. Murphy C, Schubert CR, Cruickshanks KJ Klein BEK, Klein R, Nondahl DM. Prevalence of olfactory impairment in older adults. JAMA. 2002; 288: 2307-2312.

9. White $T L$, Kurtz DB. The relationship between metacognitive awareness of olfactory ability and age in people reporting chemosensory disturbances. Am J Psychol 2003; 116: 99-110.

10. Welge-Luessen A, Hummel T, Stojan T, Wolfensberger $\mathrm{M}$. What is the correlation between ratings and measures of olfactory function in patients with olfactory loss? Am J Rhinol. 2005; 19: 567-571.

11. Shu CH, Hummel T, Lee $\mathrm{PL}$, Chiu $\mathrm{CH}_{\text {, Lin } \mathrm{SH}}$ Yuan BC. The proportion of self-rated olfactory dysfunction does not change across the life span. Am J Rhinol Allergy. 2009; 23 413-416.

12. Ritchie K, Artero S, Touchon J. Classification criteria for mild cognitive impairment: a population based validation study. Neurology. 2001; 56: 27-42.

13. Nordin S, Brämerson A. Complaints of olfactory disorders: epidemiology, assessment and clinical implications. Curr Opin Allergy Clin Immunol. 2008; 8: 10-15.

14. Croy I, Nordin S, Hummel T. Olfactory disorders and quality of life: an updated review. Chem Sens. 2014; 39: 185-194.

15. Bromley SM. Smell and taste Disorders: a primary care approach. Am Fam Physician. 2000; 61: 427-436.

16. World Medical Association. Declaration of Helsinki: Recommendations guiding physicians in biomedical research involving human subjects. JAMA. 1997; 277: 925-926.

17. Wechsler D. Wechsler Abbreviated Scales of Intelligence. New York: The Psychological Corporation, 1999.

18. Delis DC, Kramer JH, Kaplan D, Ober BA California Verbal Learning Test. 2nd ed. San Antonio, TX: Psychological Corporation, 2000

19. Delis DC, Kaplan E, Kramer JH. Delis-Kaplan Executive Function Scale. San Antonio, TX: Psychological Corporation, 2001.

20. Reitan RM, Davidson LA. Clinical neuropsychology: current status and application. New York: Winston/Wiley, 1974.

21. Heaton, RK, Miller SW, Taylor MT, Grant I. Revised comprehensive norms for an Expanded Halstead-Reitan Battery: demographically adjusted neuropsychological norms for African American and Caucasian Adults. Odessa: Psychological Assessment Resources, Inc, 2004

22. Wechsler D. Manual for the Wechsler Adult Intelligence Scale-Revised. New York: Psychological Corporation, 1981.

23. Petersen RC, Smith GE, Waring SC, Ivnik RJ, Tangalos EG, Kokmen E. Mild cognitive impairment: clinical characterization and outcome. Arch Neurol. 1999; 56: 303-308.

24. Nordin S, Brämerson A, Lidén E, Bende M The Scandinavian Odor-Identification Test: development, reliability, validity, and normative data. Acta Otolaryngol. 1998; 118:
226-234

25. Murphy C, Doty RL, Duncan HJ. Clinical disorders of olfaction. In: Doty RL, ed. Handbook of olfaction and gustation. New York: Marcel Dekker, 2003; 461-478.

26. Hedén Blomqvist $E$, Brämerson A, Stjärne P, Nordin S. Consequences of olfactory loss and adopted coping strategies. Rhinology. 2004; 42: 189-194.

27. Doty RL, Shaman P, Dann M. Development of the University of Pennsylvania Smell Identification Test: a standardized microencapsulated test of olfactory function. Physiol Behav. 1984; 32: 489-502.

28. Cain WS. Testing olfaction in a clinical setting. Ear Nose Throat J. 1989; 68: 316

29. Hummel T, Sekinger B, Wolf SR, Pauli E, Kobal G. 'Sniffin' sticks': olfactory performance assessed by the combined testing of odor identification, odor discrimination and olfactory threshold. Chem Sens. 1997; 22: 39-52.

30. Saito S, Ayabe-Kanamura S, Takashima Y, et al. Development of a smell identification test using a novel stick-type odor presentation kit. Chem Sens 2006; 31: 379-391.

31. Davidson TM, Murphy C. Rapid clinical evaluation of anosmia: the alcohol sniff test Arch Otolaryngol Head Neck Surg. 1997; 123: 591-594

32. Jackman AH, Doty RL. Utility of a three-item smell identification test in detecting olfactory dysfunction. Laryngoscope. 2005; 115: 2209-2212.

33. Hummel T, Pfetzing U, Lötsch J. A short olfactory test based on the identification of three odors. J Neurol. 2010; 257: 1316-1321.

34. Landis BN, Frasnelli J, Croy I, Hummel T. Evaluating the clinical usefulness of structured questions in parosmia assessment. Laryngoscope. 2010; 120: 1707-1713.

35. Nordin S, Brämerson A, Murphy C, Bende M. A Scandinavian adaptation of the MultiClinic Smell and Taste Questionnaire: evaluation of questions about olfaction. Acta Otolaryngol. 2003; 123: 536-542. 
Steven Nordin, PhD

Department of Psychology

Umeå University

SE-901 87 Umeå

Sweden
Tel: +46-90-786 6006

E-mail: steven.nordin@psy.umu.se

\section{APPENDIX}

\section{Validity of the SOIT for a Norwegian sample}

In total, 914 individuals from Norway $(n=247)$ and Sweden ( $n=$ 667) were included for evaluation of the validity of the objective assessment of olfactory function (the SOIT) for use in a Norwegian sample. The Swedish sample included participants from the Skövde Population-Based Study ${ }^{(7)}$ who were selected to be matched for age and sex with the Norwegian sample. To evaluate the discriminative validity with respect to age-related effects, the participants were categorized into three age groups, 46-54, 55-64 and 65-79 years. Information about pairs of Norwegian and Swedish participants within each of the three age groups is presented in Table 3.

The number of normosmic, hyposmic, and functionally anosmic participants based on diagnostic cut-off scores for young adults (15-34 years) ${ }^{(24)}$ are presented in Table $4 . \mathrm{Chi}^{2}$ analyses showed no significant difference between the two countries in distribution across diagnoses for any of the three age groups
$\left[X^{2}(1)=0.01-1.44, n s ;\right.$ hyposmic and functionally anosmics were combined to avoid cell frequencies $<5$ ]. Chi $^{2}$ analyses (combining hyposmics and anosmics) yielded significant differences in distribution of diagnoses across age groups for both the Norwegian $\left[x^{2}(2)=9.81, p<0.01\right]$ and Swedish $\left[x^{2}(2)=29.88, p<0.001\right]$ samples. Further $\mathrm{chi}^{2}$ analyses showed, for both the Norwegian and Swedish sample, significant differences in diagnostic distributions between the age groups $46-54$ and $65-75$ years $\left[X^{2}(1)\right.$ $=7.79-27.42, p<0.01]$ and between the age groups 55-64 and 65-75 years $\left[x^{2}(1)=4.37-12.84, p<0.05\right]$, but not between the age groups 46-54 and 55-64 years $\left[x^{2}(1)=1.39-2.55\right.$, ns]. Based on the assumption that Norwegians and Swedes do not differ in olfactory function, the lack of significant difference between the two countries provides support for the SOIT and its cut-off scores being valid for use also for Norwegian populations. The differences in diagnostic frequencies between the Norwegian age groups suggest good discriminative validity of the SOIT for use in Norway.

Table 3. Number of Norwegian and Swedish participants and mean \pm SD age for different age groups for evaluation of validity of the SOIT for Norwegian use.

\begin{tabular}{|c|c|c|c|c|c|c|c|c|}
\hline \multirow{3}{*}{$\begin{array}{c}\text { Age group } \\
\text { (years) }\end{array}$} & \multicolumn{4}{|c|}{ Number of participants } & \multicolumn{4}{|c|}{ Age (years) } \\
\hline & \multicolumn{2}{|c|}{ Women } & \multicolumn{2}{|c|}{ Men } & \multicolumn{2}{|c|}{ Women } & \multicolumn{2}{|c|}{ Men } \\
\hline & Norwegian & Swedish & Norwegian & Swedish & Norwegian & Swedish & Norwegian & Swedish \\
\hline $46-54$ & 31 & 134 & 15 & 137 & $50.0 \pm 2.8$ & $50.4 \pm 2.7$ & $50.1 \pm 2.6$ & $49.9 \pm 3.1$ \\
\hline $55-64$ & 57 & 124 & 27 & 103 & $59.5 \pm 2.6$ & $59.4 \pm 2.6$ & $59.3 \pm 2.4$ & $59.6 \pm 2.7$ \\
\hline $65-79$ & 81 & 85 & 36 & 84 & $68.9 \pm 2.7$ & $69.6 \pm 2.8$ & $70.6 \pm 3.4$ & $69.4 \pm 2.7$ \\
\hline
\end{tabular}

Table 4. Number (and percentages) of normosmic, hyposmic, and functionally anosmic Norwegian and Swedish participants for age group on diagnostic cut-off scores for young adults for evaluation of validity of the SOIT for Norwegian use.

\begin{tabular}{|c|c|c|c|c|c|c|}
\hline $\begin{array}{l}\text { Age group } \\
\text { (years) }\end{array}$ & Normosmic & Norwegian & Anosmic & Normosmic & $\begin{array}{l}\text { Swedish } \\
\text { Hyposmic }\end{array}$ & Anosmic \\
\hline $46-54$ & 40 (87\%) & $5(11 \%)$ & $1(2 \%)$ & 235 (87\%) & $24(9 \%)$ & $12(4 \%)$ \\
\hline $55-64$ & $66(79 \%)$ & 16 (19\%) & $2(2 \%)$ & 185 (81\%) & 33 (15\%) & $9(4 \%)$ \\
\hline $65-79$ & $76(65 \%)$ & $36(31 \%)$ & $5(4 \%)$ & 111 (66\%) & $42(25 \%)$ & $16(9 \%)$ \\
\hline
\end{tabular}

